\title{
Incidencia de hipocalcemia pos tiroidectomía total
}

\author{
Patricio Gac E, Patricio Cabané T, José Amat V, Felipe \\ H uidobro Ga, Ricardo Rossi F, Francisco Rodríguez F, \\ Carlos Ferrada V, Francisco Cardemil R.
Incidence of hypocalcemia after total thyroidectomy

Background: Postoperative hypocalcemia is one of the most common complications of thyroid surgery. It is related to the type of disease (malignant or benign), the number of identified parathyroid glands during the surgical procedure, and the surgeon's experience. Total thyroidectomy is the procedure of choice in our hospital for benign and malignant thyroid disease, but it can increase the incidence of complications. Aim: To evaluate the incidence of postoperative hypocalcemia in patients subjected to a total thyroidectomy. Material and methods: Two studies were performed. A retrospective review of medical records of 448 patients subjected to total thyroidectomy, looking for serum calcium levels of less than $8 \mathrm{mg} / \mathrm{dl}$ and clinical signs of hypocalcemia. In a second study, 45 patients were followed with measurements of preoperative and postoperative serum calcium levels. Results: In the retrospective study, only 136 records had reliable information. Clinical signs of hypocalcemia were registered in $14 \%$ of patients and a low serum calcium level was detected in $50 \%$. In the prospective study, $42 \%$ of patients had a postoperative low serum calcium level and seven patients (15\%) had symptoms. Patients were handled with oral calcium and calcitriol in some cases. Ninety nine percent of patients had normal serum calcium levels two moths after surgery. Conclusions: In this series, the rate of postoperative hypocalcemia after total thyroidectomy is similar to internaitonal reports (Rev Méd Chile 2007; 135: 26-30).

(Key words: Hypocalcemia; Thyroidectomy; Thyroid diseases)

Recibido el 20 de enero, 2006. Aceptado el 14 de julio, 2006.

Departamento de Cirugía Endocrina y General, Hospital Clínico Universidad de Chile.

aMédico en Programa de Formación de Especialista en Cirugía, Universidad de Chile.

$\mathrm{U}$ na de las complicaciones más frecuentes de la cirugía tiroidea es la hipocalcemia posoperatoria, a causa de un hipoparatiroidismo posquirúrgico, transitorio o permanente, principalmente

Correspondencia a: Dr. Patricio Gac E. Santos Dumont 999. Teléfonos: 9788000-2014607. E mail: patriciogac@yahoo.com.ar debido a compromiso de la vascularización de las glándulas paratiroides o a la resección inadvertida de éstas.

Los factores de riesgo descritos de hipoparatiroidismo posquirúrgico permanente en cirugía tiroidea son: extensión de la resección, disección ganglionar del grupo VI, tiroidectomía como terapia de hipertiroidismo, ligadura central (próximal) de la arteria tiroidea inferior, número de 
paratiroides identificadas y preservadas en el acto operatorio y la experiencia del cirujano ${ }^{1}$.

Por lo tanto, se recomienda que en cirugía bilateral de tiroides se realice ligadura periférica de la arteria tiroidea inferior (cercana a la cápsula tiroidea) y se preserven al menos 2 glándulas paratiroides (no se reporta beneficio si se preserva un número mayor) ${ }^{2}$.

El hipoparatiroidismo transitorio (menor a 6-12 meses) ocurre en un rango de $6,9 \%$ a $46 \%$ de las tiroidectomías. El hipoparatiroidismo posquirúrgico permanente ( $>6$ a 12 meses) ocurre en $0,4 \%$ a $33 \%$ de las cirugías de tiroides ${ }^{1,3}$.

Las consecuencias de esta patología son: parestesias, calambres e incluso tetania. A largo plazo, calcificación de los ganglios basales del cerebro, mayor riesgo de formación de cataratas y, finalmente, el alto costo involucrado en el tratamiento definitivo ${ }^{4}$.

En nuestro centro, al igual que en la mayoría de los centros nacionales e internacionales, la tiroidectomía total (TT), o casi total, ha reemplazado gradualmente a cirugías parciales o subtotales en el manejo de enfermedades benignas y malignas del tiroides. Esta conducta se ha implementado debido a la posibilidad de multifocalidad en cáncer de tiroides, recidiva de nódulos en lóbulo remanente en patología benigna y edades de presentación temprana de la patología tiroidea maligna; además, en cáncer de tiroides, la TT tiene mejor resultado en adyuvancia con radioyodo, mejor posibilidad de seguimiento con tiroglobulina, junto a baja morbimortalidad de nuestro grupo. Sin embargo, la TT podría aumentar la incidencia de hipocalcemia posoperatoria.

El objetivo del presente trabajo fue evaluar la incidencia y factores de riesgo de hipocalcemia en casos de tiroidectomía total en el Hospital Clínico de la Universidad de Chile.

\section{SujETOS Y MÉTODO}

Se diseñaron 2 tipos de estudio. Uno retrospectivo, en que se revisaron fichas de 448 pacientes sometidos a tiroidectomía total (TT), obtenidas del registro de estadística del Hospital Clínico de la Universidad de Chile, entre 1992 y 2002. Se analizó la incidencia de hipocalcemia diagnosticada por laboratorio $(<8,0 \mathrm{mg} / \mathrm{dl})$ y por clínica (parestesias y presencia del signo de Chvostek) en relación a edad, sexo, tipo de cirugía (total 0 parcial), diagnóstico posoperatorio $\mathrm{y}$, en caso de cáncer de tiroides, compromiso ganglionar al momento de la cirugía. Se excluyeron pacientes sin antecedentes completos en la ficha clínica y con seguimiento menor a 1 mes. El otro diseño fue un seguimiento prospectivo entre mayo y agosto de 2004 (45 pacientes). Se midió calcemia preoperatoria, 24 y $48 \mathrm{~h}$ posoperatorias (en laboratorio central por método automatizado, cresolftaleína). A los pacientes que desarrollaron hipocalcemia se les controló calcemia semanalmente. Se documentó la impresión clínica subjetiva del cirujano en relación a la viabilidad de las glándulas paratiroides al término de la operación. El punto de corte para definir hipocalcemia fue también de $8,0 \mathrm{mg} / \mathrm{dl}$. Se definió hipocalcemia transitoria a aquella menor a 6 meses de duración.

Los resultados se expresan como porcentajes y se estableció las diferencias estadísticas por método de comparación de proporciones con el software Statistical Primer of Biostatistics 3.0.

\section{Resultados}

En el estudio retrospectivo se logró obtener datos fidedignos y seguimiento mayor a 1 mes en 136 casos de TT. El seguimiento promedio fue de 18,6 meses (mínimo 6 meses y máximo 156 meses). La edad promedio de los pacientes fue de 45,8 años. El $79,3 \%$ de los casos correspondió a mujeres y $20,7 \%$ a hombres. La mayor incidencia de hipocalcemia fue en el rango de 30-49 años. El 89\% de las tiroidectomías totales se realizaron por cáncer tiroideo. Hubo hipocalcemia en $23,7 \%$ de las cirugías por cáncer papilar y en 25\% por cáncer folicular. Tanto la presencia de metástasis cervicales, como la necesidad de reoperación (7\%), no se relacionaron con mayor incidencia de hipocalcemia en esta serie, salvo para los casos de hipocalcemia permanente. $\mathrm{Si}$ el diagnóstico de hipocalcemia se basaba en la clínica (presencia de parestesias, signo de Chvostek), se pesquisó en 14,8\% de las cirugías. En cambio, si el diagnóstico se realizó por control de calcemia seriado se obtuvo hasta $50 \%$ de hipocalcemia. El 40\% de estos casos se recuperó en menos de $24 \mathrm{~h}$ con aporte de calcio oral, en dosis de 0,5 gr a 1 gr c/6-8 h. Se registró sólo 2 casos de 
hipoparatiroidismo permanente (1,5\%), que comespondió a un paciente reoperado por recivida de cáncer papilar y a un paciente con tiroiditis de Hashimoto. No se describieron secuelas a lango plazo en ninguno de los casos revisados.

El estudio prospectivo reveló que en 45 TT realizadas en el período indicado, las calcemias posoperatorias variaron entre 6,8 y $9,3 \mathrm{mg} / \mathrm{dl}(8,1$ $\mathrm{mg} / \mathrm{dl}$ promedio). Hubo 19 pacientes (42\%) con calcemia menor a $8 \mathrm{mg} / \mathrm{dl}$, de los cuales 17 (84\%) se produjeron a las $24 \mathrm{~h}$ y $2(16 \%)$ a las $48 \mathrm{~h}$. Se describió vitalidad de las 4 paratiroides en 42 pacientes, de los cuales 14 presentaron hipocalcemia (33\%). En 2 pacientes se describió la vitalidad como dudosa de una de las paratiroides y en 1 paciente fue necesario autoinjertar una, todos estos casos presentaron hipocalcemia. En 18 pacientes en quienes se midió calcemia preoperatoria, ésta descendió en todos los casos, con una baja que varió entre 0,2 y $2,2 \mathrm{mg} / \mathrm{dl}$, con un promedio de $1,32 \mathrm{mg} / \mathrm{dl}$. Siete pacientes (15\%) presentaron sintomatología (principalmente parestesias), Chvostek positivo o ambos, que fue muy notoria (grado III) en 2 (4\%) y leve (grado I y II) en $5(11 \%)$. No hubo casos con tetania. Todos los pacientes con calcemia menor a 8 recibieron tratamiento con lactogluconato más carbonato de calcio oral (1/2 gr a $1 \mathrm{gr} \mathrm{c} / 6-8 \mathrm{~h})$ y asociado en ocasiones a calcitriol (calcemia $<7,0 \mathrm{mg} / \mathrm{dl} \quad 0$ síntomas severos); de estos casos de hipocalcemia, 6 (32\%) se normalizaron en 24 h, 10 (52\%) en 1 semana y 3 (16\%) en más de 1 semana. Durante el control semanal posoperatorio se logró ir disminuyendo el aporte oral de calcio, hasta suspenderlo. El 99\% de los pacientes normalizó sus calcemias a los 2 meses de control. En este grupo no hubo hipoparatiroidismo definitivo y en ningún paciente fue necesario terapia con calcio endovenoso.

Al comparar los resultados del estudio retrospectivo versus el prospectivo, no se encontraron diferencias estadísticamente significativas en cuanto a incidencia de hipocalcemia transitoria 0 permanente, y recuperación de esta (Tabla 1).

\section{Discusión}

En Chile existe escasa información sobre la incidencia de hipocalcemia posterior a cirugía tiroidea, ella está limitada a revisiones retrospectivas de cirugía de cáncer de tiroides $^{5}$ y reportes personales ${ }^{6}$, entre otros.

Es importante notar que entre $42 \%$ y $50 \%$ de nuestros enfermos presentan algún grado de hipocalcemia transitoria, si se diagnostica por laboratorio, de las cuales, una gran mayoría son asintomáticas y no relevantes desde el punto de vista pronóstico. En la literatura se comunica un rango muy variable de $6,9 \%$ a $46 \%$ de hipocalcemia postiroidectomía, lo que podría explicarse por el método de pesquisa (sólo clínica, laboratorio o ambos); si se analiza esta serie, sólo 15\% de los pacientes fueron sintomáticos. Encontramos 2 pa-

\section{Tabla 1. Comparación de resultados de hipocalcemia postiroidectomía total en estudio retrospectivo versus prospectivo}

\begin{tabular}{|lcccc|}
\hline & $\begin{array}{c}\text { Retrospectivo } \\
1992-2002\end{array}$ & $\begin{array}{c}\text { Prospectivo } \\
\text { V-VIII/2004 }\end{array}$ & $\mathrm{p}$ & \\
\hline Hipocalcemia transitoria & $14,8 \%$ & $15 \%$ & 0,8 & NS \\
- por clínica & $50 \%$ & $42 \%$ & 0,4 & NS \\
- por laboratorio & $1,5 \%$ & $0 \%$ & 0,8 & NS \\
Hipocalcemia permanente & & & & NS \\
Recuperación & $40 \%$ & $32 \%$ & 0,3 & \\
$\quad$ 24 h & & $52 \%$ & & \\
1 semana & & $16 \%$ & & \\
N 1 semana & 136 & 45 & & \\
\hline
\end{tabular}


cientes con hipocalcemia definitiva, uno operado por recidiva tumoral y otro con tiroiditis crónica de base, factores que probablemente aumentan el riesgo de hipoparatiroidismo posquirúrgico, por el cambio anatómico que conlleva el primero, y por la aumentada vascularización y fibrosis en el caso de la tiroiditis, lo que, a nuestro parecer, dificulta la identificación de las glándulas paratiroides.

Diversos centros prefieren el tratamiento rutinario con calcio, o incluso calcitriol, en el posoperatorio de tiroidectomía total, con el objeto de evitar precozmente la sintomatología y el riesgo de tetania. Es así como un reciente estudio prospectivo y aleatorizado señala que el aporte de $500 \mathrm{mg}$ de calcio c/8 h p.o, más $1 \mu \mathrm{g}$ de calcitriol al día (en dos tomas) reduce significativamente la incidencia de tetania. Por otro lado, describen que la mayor severidad de la hipocalcemia es al tercer día, cuando habitualmente los pacientes son dados de alta, por lo que han instaurado el uso normado de calcio en el posoperatorio ${ }^{7}$. En nuestro estudio, la mayor incidencia de hipocalcemia fue en las primeras 24-48 $\mathrm{h}$, pero no fue posible analizar el momento de mayor severidad de ésta, pues, habitualmente, con el diagnóstico de hipocalcemia se inició tratamiento, con el fin de evitar sintomatología.

El hallazgo de un importante porcentaje de hipocalcemia de laboratorio (asintomáticas), creemos que es multifactorial y no necesariamente secundario sólo a hipoparatiroidismo; por ejemplo, la hemodilución intra y posoperatoria podría disminuir el valor de la calcemia; el correcto manejo de la muestra desde su toma (muestra periférica, lejos de infusiones endovenosas), su traslado (mantención de cadena de frío) y el método de análisis del $\mathrm{Ca}^{+2}$ plasmático (calibración adecuada de la máquina). No consideramos necesaria la corrección de calcemia por albúmina plasmática, ya que se ha reportado un descenso de ésta en correlación con la calcemia en pacientes sin comorbilidad 8,9 .

Por otro lado, la clínica (reflejada en parestesias y signos específicos) parece ser poco sensible en comparación al laboratorio.

En este estudio, además se consideró la impresión subjetiva de la vitalidad de la paratiroides en el intraoperatorio, que resultó ser un predictor limitado del riesgo de hipocalcemia. A pesar de la visión directa y certeza subjetiva de la vitalidad de la paratiroides por parte del cirujano, un tercio de estos pacientes presentó hipocalcemia transitoria en el posoperatorio.

De nuestra casuística puede concluirse que la ausencia de síntomas específicos o la impresión subjetiva de vitalidad de las glándulas paratimoides en el intraoperatorio no descarta la presencia de hipocalcemia, por lo cual la clínica sola no puede guiar nuestra conducta a este respecto. Así, considerando que por laboratorio existe un sobrediagnóstico de hipocalcemias y siendo la clínica poco sensible, hemos optado por solicitar de regla una calcemia a las $24 \mathrm{~h}$ posoperatorias. Si ésta es normal y el paciente asintomático no recibe terapia con calcio; si la calcemia está bajo $8,0 \mathrm{mg} / \mathrm{dl}$, independientemente si el paciente es asintomático o sintomático leve, le indicamos lactogluconato de calcio 0,5 a $1 \mathrm{~g}$ c/8 h p.o. Agregamos calcitriol en pacientes sintomáticos severos o con calcemia menor a 7,0 mg/dl. Sólo utilizamos calcio endovenoso en los pacientes con tetania.

Durante el control de calcemia semanal, 99\% de los pacientes normaliza las calcemias y suspende el aporte oral de calcio a los 2 meses; por esto creemos que luego de este período, los pacientes que persisten con hipocalcemia y requerimiento de calcio oral en dosis elevadas forman parte del grupo de riesgo de hipoparatiroidismo permanente.

Consideramos que el uso de calcio posoperatorio normado es una medida sencilla, de bajo costo y que se logra suspender a corto plazo. Evita la aparición de síntomas en pacientes que podrían evolucionar con sintomatología leve y además aporta mayor seguridad al paciente, sobre todo cuando habita en sectores alejados de un centro de atención de salud.

En conclusión, en pacientes sometidos a tiroidectomía total, encontramos hipocalcemia posoperatoria hasta en $50 \%$ de los casos, la mayor parte asintomáticos u oligosintomáticos. La inmensa mayoría de los casos normaliza su calcemia antes de dos meses y sólo 1,5\% queda con hipocalcemia definitiva. 


\section{REFERENCIAS}

1. Thomusch O, Machens A, Sekula $C$, Ukkat J, BraucKHoFf M, Draue H. The impact of surgical technique on postoperative hypoparathyroidism in bilateral thyroid surgery: A multivariate analysis of 5846 consecutive patients. Surgery 2003; 133: 180-5.

2. Ríos A, Rodríguez JM, Canteras $M$, Riquelme J, Iliana J, Balsalobre MD, Parrila P. Estudio multivariable de los factores de riesgo para desarrollar complicaciones en la cirugía del bocio multinodular. Cir Esp 2005; 77: 79-85.

3. Снош TL, Cнu W, Lм BH, Kwок SPY. Outcomes and complications of thyroid surgery: retrospective study. HKMJ 2001; 7: 261-5.

4. Minai R, Farndon JR. Parathyroid disease and calcium metabolism. Br J Anaesth 2000; 85: 29-43.

5. González M, Andrades P, Contreras O, Irarrázabal V, Paladines P, Inturias R et al. Sobrevida en cáncer de tiroides: seguimiento de 365 pacientes por un período de 37 años. Rev Chil Cir 2002; 54: 329-35.

6. Lobos Martínez A. Tratamiento quirúrgico del cáncer de tiroides: experiencia personal. Rev Chil Cir 2002; 54: 485-90.

7. Tartagla F, Giuliani A, Sgueglia M, Biancari $F$, Juvonen T, Campana FP. Randomized study on oral administration of calcitriol to prevent symptomatic hypocalcemia after total thyroidectomy. Am J Surgery 2005; 190: 424-9.

8. Cakmakli S, Cavusoglu T, Bumin C, Torun N. Postthyroidectomy hypocalcemia: the role of calcitonin, parathormone and serum albumin. Tokai J Exp Clin Med 1996; 21: 97-101.

9. Demeester-Mirkine N, Hooghe L, Van Geertruyden J, De Maertelaer V. Hypocalcemia after thyroidectomy. Arch Surg 1992; 127: 854-8. 